\title{
Hydrogen Sulfide Protects Cardiomyocytes from Myocardial Ischemia-Reperfusion Injury by Enhancing Phosphorylation of Apoptosis Repressor with Caspase Recruitment Domain
}

\author{
Xiaoyi Yao, ${ }^{1, *}$ Gang Tan, ${ }^{2, *}$ Changjun He, ${ }^{2}$ Yan Gao, ${ }^{3}$ Shangha Pan, ${ }^{2}$ \\ Hongchi Jiang, ${ }^{2}$ Yina Zhang ${ }^{1}$ and Xueying Sun ${ }^{2}$ \\ ${ }^{1}$ Department of Geriatrics, The Second Affiliated Hospital of Harbin Medical University, Harbin, P.R. China \\ ${ }^{2}$ The Hepatosplenic Surgery Center, The First Affiliated Hospital of Harbin Medical University, Harbin, P.R. \\ China \\ ${ }^{3}$ Department of ICU, The Fourth Affiliated Hospital of Harbin Medical University, Harbin, P.R. China
}

\begin{abstract}
Hydrogen sulfide $\left(\mathrm{H}_{2} \mathrm{~S}\right)$ displays an anti-apoptotic activity against myocardial ischemia reperfusion (MIR). Apoptosis repressor with caspase recruitment domain (ARC) is constitutively expressed in the heart and inhibits cell apoptosis when it is phosphorylated. Here, we investigated whether $\mathrm{H}_{2} \mathrm{~S}$ could inhibit apoptosis by affecting ARC phosphorylation using cultured rat cardiomyocytes and a rat model of MIR. Primary cardiomyocytes were prepared from hearts of newborn rats and were pre-incubated with NaHS, a donor of $\mathrm{H}_{2} \mathrm{~S}$, for 60 min. Cardiomyocytes were subjected to hypoxia for $4 \mathrm{~h}$, followed by reoxygenation for $2 \mathrm{~h}$. The hypoxia and subsequent reoxygenation $(H / R)$ significantly induced cell apoptosis, increased expression levels of Fas and FasL proteins, enhanced release of cytochrome $c$ from mitochondria, and elevated caspase- 3 activity, while H/R reduced ARC phosphorylation and increased the activity of calcineurin that dephosphorylates ARC. Pre-incubation with NaHS significantly attenuated the above effects through promoting ARC phosphorylation by reducing calcineurin activity and by increasing the activity of protein kinase casein kinase II (CK2) that phosphorylates ARC. In fact, TBB, a specific inhibitor of CK2, abolished the effects of NaHS. In rats undergoing MIR, NaHS significantly reduced the myocardial infarct size, cell apoptosis, calcineurin activity, and the expression levels of Fas, FasL and cleaved caspase-3 proteins, while NaHS increased ARC phosphorylation. In contrast, DL-propargylglycine, an inhibitor of cystathionine $\gamma$-lyase, the main enzyme for $\mathrm{H}_{2} \mathrm{~S}$ production in hearts, showed opposite effects to NaHS. The results indicate that $\mathrm{H}_{2} \mathrm{~S}$ inhibits apoptosis of cardiomyocytes induced by MIR through enhancing ARC phosphorylation.
\end{abstract}

Keywords: apoptosis; apoptosis repressor with caspase recruitment domain; cardiomyocytes; hydrogen sulfide; myocardial ischemia reperfusion

Tohoku J. Exp. Med., 2012, 226 (4), 275-285. C 2012 Tohoku University Medical Press

Hydrogen sulfide $\left(\mathrm{H}_{2} \mathrm{~S}\right)$ is the third gaseous mediator and exerts many physiological and pathological activities (Elrod et al. 2007). $\mathrm{H}_{2} \mathrm{~S}$ is endogenously produced from L-cysteine by cystathionine $\beta$-synthase (CBS) and cystathionine $\gamma$-lyase (CSE) (£owicka and Betowski 2007), and the production of endogenous $\mathrm{H}_{2} \mathrm{~S}$ in hearts is mainly by CSE (Chen et al. 1999). $\mathrm{H}_{2} \mathrm{~S}$ has shown protective roles in myocardial ischemia reperfusion (MIR)-induced injury (Pan et al. 2006; Elrod et al. 2007; Bliksøen et al. 2008; Yong et al. 2008; Sivarajah et al. 2009). The anti-apoptotic activity of $\mathrm{H}_{2} \mathrm{~S}$ is increasingly important for the cardioprotection against MIR as it regulates factors in both death-receptor and mitochondria apoptotic pathways (Pan et al. 2006;
Elrod et al. 2007). We have previously reported that $\mathrm{H}_{2} \mathrm{~S}$ protected hearts from MIR-induced injury in diabetic rats by its anti-apoptotic activity via downregulating expression of active caspase-3, Fas, FasL and TNF- $\alpha$ (Gao et al. 2011).

Apoptosis repressor with caspase recruitment domain (ARC) is constitutively expressed in the myocardium, and it acts to inhibit apoptosis through both intrinsic and extrinsic apoptotic pathways (Nam et al. 2004; Ekhterae et al. 2011). Overexpression of ARC inhibited ischemia-induced apoptosis of $\mathrm{H} 9 \mathrm{c} 2$ cardiomyoblast cells by preventing mitochondrial cytochrome $c$ release (Ekhterae et al. 1999). Transduction of ARC reduced infarct size of isolated rat hearts after MIR and blocked the development of cardiomy-

Received January 11, 2012; revision accepted for publication March 16, 2012. doi: 10.1620/tjem.226.275

*Xiaoyi Yao and Gang Tan contributed equally to this work.

Correspondence: Yina Zhang, M.D., Department of Geriatrics, the Second Affiliated Hospital of Harbin Medical University, 150086 Harbin, P.R. China.

e-mail: yinazhanghmu@126.com. 
opathy (Gustafsson et al. 2002). ARC knockout mice exhibited enhanced sensitivity to hypoxic injury in hearts (Donath et al. 2006), and ARC contributed to the inhibitory effect of preconditioning on cardiomyocyte apoptosis ( $\mathrm{Li}$ et al. 2007). ARC phosphorylation is essential for its activation, as only phosphorylated ARC is able to translocate from the cytoplasm to the mitochondria to exert its antiapoptotic activity ( $\mathrm{Li}$ et al. 2002; Tan et al. 2008).

However, it is not known whether $\mathrm{H}_{2} \mathrm{~S}$ could inhibit cardiomyocyte apoptosis induced by MIR through its effects on ARC phosphorylation. Therefore, we designed this study to examine the possible role of ARC phosphorylation in the inhibitory effects of $\mathrm{H}_{2} \mathrm{~S}$ on cardiomyocyte apoptosis in an in vitro model of hypoxia/reoxygenation (H/ $\mathrm{R}$ ) using primary rat cardiomyocytes and a rat model of MIR.

\section{Materials and Methods}

Animals, reagents and antibodies

Wistar rats were supplied by the Animal Research Center at the Second Affiliated Hospital of Harbin Medical University, Harbin, P.R. China. All the procedures administered to the animals were in compliance with the institutional guidelines. Sodium hydrosulfide (NaHS) (the donor of $\mathrm{H}_{2} \mathrm{~S}$ ), DL-propargylglycine (PAG) (an irreversible inhibitor of CSE (Gao et al. 2011), and 4,5,6,7-tetrabromobenzotriazole (TBB) (Lu et al. 2011) were purchased from SigmaAldrich. Antibodies used in this study included anti-cardiac $\alpha$-sarcomeric actin $\mathrm{Ab}$ (Sigma-Aldrich), Abs against Fas, FasL, cleaved caspase-3, voltage dependent anion channel (VDAC) and Rho GDP-dissociation inhibitor (RhoGDI) (Santa Cruz Biotechnology, Santa Cruz, CA, USA), anti-ARC Ab (Chemicon International Inc, MA, USA), anti-phospho Thr-149 Ab (Eurogentec, Seraing, Belgium), anti-cytochrome $c$ Ab (BD Pharmagin, San Jose, CA, USA).

\section{Preparation and culture of primary rat cardiomyocytes}

The methodology has been described previously (Shiraishi et al. 2001). Briefly, the heart dissected from 1 to 3-day-old male rats was washed with ice-cold Hanks' balanced salt solution (BSS), and then cardiomyocytes were dissociated with $0.15 \%$ trypsin in BSS for 20 min for 3 times. Each time, the cell suspension was centrifuged at $1,000 \times g$, and the cell pellet was washed with DMEM (Gibco, Carlsbad, CA, USA) supplemented with $10 \%$ fetal calf serum (FCS). The cells were cultured at $37^{\circ} \mathrm{C}$ under a water-saturated atmosphere of $5 \% \mathrm{CO}_{2}-95 \%$ air for $72 \mathrm{~h}$ prior to experiments. The purity of cardiomyocytes was monitored by staining the cells with an $\mathrm{Ab}$ against cardiac $\alpha$-sarcomeric actin, and was found to be $95 \pm 3 \%$ when examined at $72 \mathrm{~h}$ after culturing.

\section{Cell hypoxia/reoxygenation $(H / R)$}

The methodology of stimulating cell $\mathrm{H} / \mathrm{R}$ in vitro has been described previously (Elrod et al. 2007). For hypoxia, cardiomyocytes were incubated in a solution $(\mathrm{pH} 6.8$ ) containing (in mmol/L) $5.37 \mathrm{KCl}, 0.44 \mathrm{KH}_{2} \mathrm{PO}_{4}, 136.89 \mathrm{NaCl}, 4.166 \mathrm{NaHCO}_{3}, 0.338$ $\mathrm{Na}_{2} \mathrm{HPO}_{4}$ and 5 glucose under an atmosphere of water-saturated $1 \%$ $\mathrm{O} 2,5 \% \mathrm{CO}_{2}$ and $94 \% \mathrm{~N}_{2}$ for $4 \mathrm{~h}$. For reoxygenation, the solution was replaced with fresh DMEM supplemented with $20 \%$ FCS, and cells were further cultured under a water-saturated atmosphere of $5 \% \mathrm{CO}_{2}$ and $95 \%$ air for $2 \mathrm{~h}$.

Cell viability assay

Cell viability was measured with an MTT kit (Shanghai Huashun Bioengineering Co., China). The cell viability index was calculated according to the formula below: experimental OD value/ control OD value $\times 100 \%$.

Lactate dehydrogenase assay

The activity of lactate dehydrogenase (LDH) released from the cells into the culture media was determined with a LDH kit (Nanjing Jianchen Bioengineering Institute, Nanjing, China) according to the manufacturer's manual.

In vitro cell apoptosis assay

Cell apoptosis was identified by staining cells with Annexin V and propidium iodide (PI) according to the manufacturer's instruction (BD Biosciences, San Jose, CA, USA). Briefly, cells were suspended in $100 \mu 1$ binding buffer, $5 \mu \mathrm{l}$ of Annexin $\mathrm{V}$ and $5 \mu \mathrm{l}$ of PI were added, and incubated for $15 \mathrm{~min}$ at room temperature in dark. The stained cells were subjected to flow cytometry to measure the apoptosis rate (\%) with a Beckman Coulter Epics Altra II cytometer (Beckman Coulter, California, USA). The cells were further stained with TUNEL (terminal deoxynucleotidyl transferase dUTP nick end labeling) (Roche Shanghai, China) according to the manufacturer's manual, and examined by fluorescence microscopy.

Caspase-3 activity assay

A kit of Caspase-Glo® 3/7 Assay (Promega, Beijing, China) was used to measure caspase-3 activity according to the manufacturer's manual.

Assay for mitochondrial cytochrome c release

Cells were incubated in lysis buffer (in mmol/L: 20 HEPES, $\mathrm{pH}$ 7.5, $10 \mathrm{KCl}, 1.5 \mathrm{MgCl}_{2}, 1.0$ sodium EDTA, 1.0 sodium EGTA, 1.0 dithiothreitol, 0.1 phenylmethylsulfonyl fluoride and 250 sucrose) for $15 \mathrm{~min}$ on ice, and then homogenized. The nuclei and cell debris were removed by centrifugation at $1,000 \times g$ for $15 \mathrm{~min}$ at $4^{\circ} \mathrm{C}$. The supernatants were centrifuged at $10,000 \times g$ for $15 \mathrm{~min}$ at $4^{\circ} \mathrm{C}$, and the resulting mitochondria pellets were resuspended with the lysis buffer. The supernatants were further centrifuged at $100,000 \times g$ for $1 \mathrm{~h}$ at $4^{\circ} \mathrm{C}$. The protein content from the cytosol and mitochondrial fractions was determined with a Pierce BCA Protein Assay Kit (Pierce Biotechnology, IL, USA), and samples subjected to Western blot analysis with an anti-cytochrome $c \mathrm{Ab}$, and with Abs against VDAC and RhoGDI to confirm the purity of mitochondrial and cytosolic fractions, respectively.

\section{Measurement of calcineurin activity}

The cells were washed with ice-cold PBS for 3 times, and lysed in $1 \mathrm{mM}$ phenylmethylsulfonyl fluoride solution for $30 \mathrm{~min}$, followed by removal of the cell debris by centrifugation at $10,000 \times g$ for 10 min at $4^{\circ} \mathrm{C}$. Or frozen tissues were homogenized in ice-cold lysis buffer and centrifuged at $10,000 \times g$ for $45 \mathrm{~min}$ at $4^{\circ} \mathrm{C}$. Calcineurin activity in the supernatants was determined with a Calcineurin kit (Nanjing Jianchen Bioengineering Institute, Nanjing, China) according to the manufacturer's manual. 
Transfection of calcineurin SiRNA and detection of mRNA by RT-PCR

A double-strand siRNA (sense 5'-AAGCUCCAAUUACAG UGUGUGCC-3' and antisense 5'-GGCACACACUGUAAU UGGAGCUU-3') targeting nucleotides 311-331 of rat calcineurin A- $\beta$ subunit [GenBank: M31809.1] with two introduced thymidine residues at the 3' end was designed (Prasad and Inesi 2011), and produced by GenePharma Co., Ltd., Shanghai, China. A nonspecific siRNA (sense 5'-UUCUCCGAACGUGUCACGU-3' and antisense 5'-ACGUGACACGUUCGGAGAA-3') served as control. Cells were incubated with siRNAs $(0.1 \mu \mathrm{M})$ using Lipofectamine ${ }^{\mathrm{TM}} 2000$ (Invitrogen) in the serum-free medium for $24 \mathrm{~h}$, and the expression of calcineurin A- $\beta$ mRNA was detected by RT-PCR with one pair of primers (5'-TGAACACCGCACATACCACTG-3' and 5'-GCCCTC AAGCCTCCATCCG-3'), and normalized with rat GAPDH mRNA amplified with a pair of primers (5'-GTTCCAGAGACAGCC GCATC-3' and 5'-CGTTCACACCGACCTTCACC-3') (Prasad and Inesi 2011).

\section{Determination of protein kinase casein kinase II (CK2) Activity}

CK2 activity was determined by employing a CK2 activity kit (Beijing B\&M Biotech Co., Ltd. Beijing, China.). Cells were suspended in extraction buffer and measurement of CK2 activity in whole cell lysate was carried out according to the manufacturer's manual.

\section{Animal experimental design}

Forty-eight male rats (250-300 g) were randomly assigned to four groups (each group had 12 rats): sham, MIR, MIR + NaHS and MIR + PAG. The rats in the sham and MIR groups received daily i.p. injection of $1 \mathrm{ml}$ of PBS (vehicle). The rats in MIR + NaHS and MIR + PAG groups received daily i.p. injection of $1 \mathrm{ml}$ of NaHS solution (14 $\mu \mathrm{mol} / \mathrm{kg}$ ) or PAG solution $(50 \mathrm{mg} / \mathrm{kg})$, respectively. The doses were selected based on previous reports (Sivarajah et al. 2006; Kang et al. 2009; Gao et al. 2011). Seven days later, rats in the sham group underwent left thoracotomy and pericardiotomy without MIR, and rats in the other three groups underwent MIR.

\section{Surgical procedure}

The procedure has been described previously (Gao et al. 2011). Briefly, rats underwent a left thoracotomy under anesthesia. The left coronary artery was dissected and ligated just below the origin of the left circumflex artery. The artery was occluded for $30 \mathrm{~min}$, and then reperfusion started for $2 \mathrm{~h}$. The blood samples were collected via cardiac puncture, and centrifuged at 2,000 $\times g$ for $10 \mathrm{~min}$ to harvest the sera. The animals were killed by injection of $2.56 \mathrm{M} \mathrm{KCl}$ directly into the left ventricle, and hearts were immediately excised. Twelve hearts from each group were randomly divided into two groups $(n=$ 6 ), which were used to measure the infarct size or stored at $-80^{\circ} \mathrm{C}$ for the analyses below, respectively.

\section{Measurement of serum $\mathrm{H}_{2} \mathrm{~S}$}

The methods have been described previously (Kang et al. 2009). Briefly, serum was mixed with $10 \%$ trichloroacetic acid. The reaction was stopped by $1 \%$ zinc acetate, followed by incubation with N,N-dimethyl-p-phenylenediamine sulphate (DPD) for $15 \mathrm{~min}$. The absorbance at $670 \mathrm{~nm}$ was measured with a spectrophotometer. All samples were assayed in triplicate and $\mathrm{H}_{2} \mathrm{~S}$ was calculated against a calibration curve of NaHS $(0.122-250 \mu \mathrm{M})$.

\section{Quantification of infarct size}

The infarct-risk volume ratio was determined as described previously (Fishbein et al. 1981; Hausenloy et al. 2005; Gao et al. 2011). Briefly, the heart was stained by infusing $1 \mathrm{ml}$ of Evan's blue through the aorta, followed by perfusion with Krebs bicarbonate buffer to wash out unbound stains. The heart was cut into six transverse slices across the long axis, stained with 1\% 2,3,5-triphenyltetrazolium chloride (TTC) for $15 \mathrm{~min}$ at $37^{\circ} \mathrm{C}$, and then fixed in $10 \%$ formalin. The slices were photographed, and the myocardial infarct size was measured as percentage of total area, and calculated with Adobe Photoshop ( $\mathrm{CS}_{4}$, Adobe Systems Inc., San Jose, CA, USA).

\section{In situ detection of cell apoptosis}

The myocardium sections $(5 \mu \mathrm{m})$ were stained with TUNEL (Roche, Shanghai, China) according to manufacturer's instruction, and examined by light microscopy. The apoptosis index was calculated as the percentage of stained cells, namely, number of apoptotic cells $\times 100 /$ total number of nucleated cells.

\section{Western blot analysis}

The methodology has been described previously (Kang et al. 2009; Gao et al. 2011). Cells or tissues were lysed and debris was removed by centrifugation. The protein content was determined and samples were resolved on $12 \%$ polyacrylamide SDS gels, and electrophoretically transferred to polyvinylidene difluoride membranes. The membranes were blocked with 3\% BSA, incubated with primary Abs, and subsequently with secondary Abs. They were developed with 5-bromo-4-chloro-3-indolyl phosphate / nitro blue tetrazolium (Tiangen Biotech Co. Ltd., Beijing, China).

\section{Statistical analysis}

All the data are expressed as mean values \pm standard deviation (SD). A one-way analysis of variance (ANOVA) followed by the post-hoc Dunnett's test was used for evaluating statistical significance (SPSS 17.0). A value of $P<0.05$ was considered significant.

\section{Results}

\section{Cell viability and $\mathrm{LDH}$ release}

The cardiomyocytes were plated at a density of $1 \times$ $10^{3} / \mathrm{mm}^{2}$ and incubated in serum-free medium containing vehicle (PBS) or NaHS (40 $\mu \mathrm{mol} / \mathrm{L})$ for $60 \mathrm{~min}$, and then subjected to $\mathrm{H} / \mathrm{R}$. The concentration of NaHS was selected based on the previous reports (Bliksøen et al. 2008; Yao et al. 2010). As shown in Fig. 1A, H/R highly significantly ( $P$ $<0.001)$ reduced cell viability, whereas pre-incubation with $\mathrm{NaHS}$ attenuated this reduction as NaHS-treated cells had a higher viability than vehicle-treated cells $(P<0.05)$. The cell damage was further confirmed by the release of $\mathrm{LDH}$ in culture media. As shown in Fig. 1B, H/R significantly increased LDH release $(P<0.001)$, but pre-incubation with $\mathrm{NaHS}$ attenuated this increase as NaHS-treated cells released significantly $(P<0.05)$ less LDH than vehicletreated cells.

\section{NaHS inhibits cell apoptosis}

The cells in Fig. 1 were stained with Annexin V and PI, and flow cytometric analysis was used to measure the apoptosis rate. The representative histograms of flow 

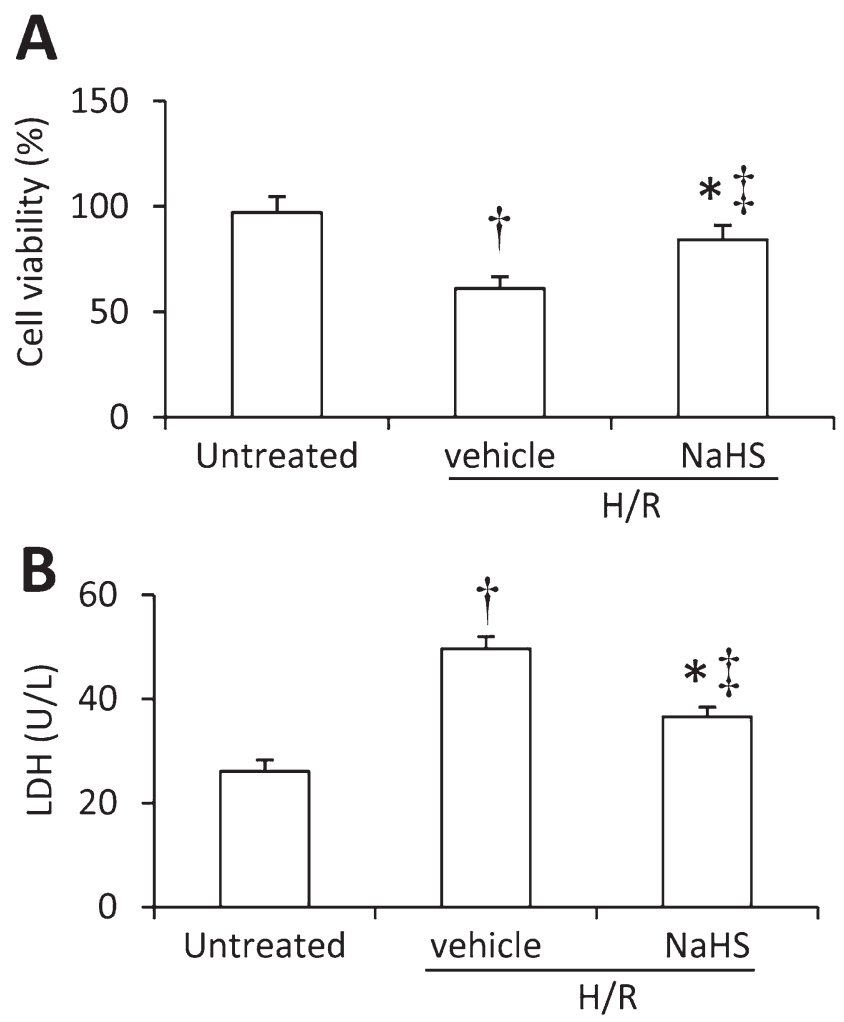

Fig. 1. Cell viability and LDH release. Cardiomyocytes were incubated with serum-free culture media containing vehicle or NaHS for $60 \mathrm{~min}$, and then subjected to hypoxia for $4 \mathrm{~h}$, followed by $2 \mathrm{~h}$ of reoxygenation (H/R). (A) Cell viability was measured. (B) The activity of LDH in culture media was measured. Compared to untreated cells, a significant difference is denoted by “*” $(P<0.05)$, and a highly significant difference by "†" $(P<0.001)$. Compared to cells subjected to $\mathrm{H} / \mathrm{R}$ and treated vehicle, a significant difference is denoted by “ $\$$ " $(P<0.05)$.

cytometry show that the apoptosis rates of untreated cells and cells subjected to $\mathrm{H} / \mathrm{R}$ and treated with vehicle or NaHS (Fig. 2A) were $3.7 \%, 30.4 \%$, or $11.3 \%$, respectively. As shown in Fig. 2B, H/R significantly increased the apoptosis rate $(P<0.001)$, whereas pre-incubation with NaHS attenuated this increase, as NaHS-treated cells had a lower apoptosis rate than vehicle-treated cells $(P<0.05)$. To further confirm cell apoptosis, the activity of caspase-3 was measured and cells were stained with TUNEL. H/R significantly increased caspase-3 activity $(P<0.001)$, but NaHS attenuated this increase, as the caspase-3 activity in NaHStreated cells was significantly $(P<0.05)$ lower than that in vehicle-treated cells (Fig. 2C). Untreated cells had sparse apoptotic cells, while $\mathrm{H} / \mathrm{R}$ induced more apoptotic cells, but pre-treatment of NaHS attenuated this increase (Fig. 2D).

We also detected cellular expression of Fas and FasL by Western blot analysis. As shown in Fig. 2E and F, H/R significantly upregulated the expression levels of Fas and FasL proteins $(P<0.05)$, whereas pre-incubation with NaHS significantly attenuated the upregulation of Fas and FasL $(P<0.05)$. We next examined the expression of cyto- chrome $c$ in cytosolic and mitochondrial fractions of the cells. Most of the cytochrome $c$ in cardiomyocytes was found in mitochondria of the untreated cells. However, H/ $\mathrm{R}$ significantly increased cytosolic concentration of cytochrome $c$ with a concomitant decrease in the mitochondria. Pre-incubation with NaHS significantly $(P<0.05)$ inhibited the release of cytochrome $c$ from mitochondria (Fig. 2E and F).

\section{NaHS increases ARC phosphorylation}

To investigate the effects of $\mathrm{H}_{2} \mathrm{~S}$ on ARC phosphorylation, the cells in Fig. 1 were used for Western blot analysis with Abs against ARC or phospho-Thr-149 (the phosphorylated form of ARC). As shown in Fig. 3A, H/R had a slight effect on the expression of total ARC, but downregulated expression of phosphorylated ARC. Pre-incubation with NaHS slightly increased expression of total ARC, but markedly upregulated expression of phosphorylated ARC, compared with vehicle. ARC phosphorylation was calculated by dividing band density of total ARC by that of phosphorylated ARC. As shown in Fig. 3B, H/R significantly $(P<$ 0.05 ) reduced ARC phosphorylation, compared with the untreated cells, but NaHS significantly increased ARC phosphorylation compared to vehicle $(P<0.05)$.

\section{NaHS inhibits calcineurin activity}

Calcineurin has been shown to dephosphorylate ARC, thus inhibiting ARC activity and promoting cell apoptosis (Tan et al. 2008). Here, we sought to determine whether NaHS influences calcineurin activity. Calcineurin activity in cells subjected to $\mathrm{H} / \mathrm{R}$ was highly significantly $(P<$ $0.001)$ higher than that in the untreated cells, but pre-incubation with NaHS significantly $(P<0.05)$ attenuated this increase (Fig. 3C). To further clarify whether NaHS increases ARC phosphorylation via its effects on calcineurin, the cells were transfected with calcineurin siRNA to silence its expression (Fig. 3D). The calcineurin-silencing cells were exposed to $\mathrm{H} / \mathrm{R}$, and treated with $\mathrm{NaHS}$. As shown in Fig. 3E and F, silencing calcineurin significantly decreased calcineurin activity and increased ARC phosphorylation, and partially abolished the effects of $\mathrm{NaHS}$ on calcineurin activity and ARC phosphorylation altered by $\mathrm{H} /$ $\mathrm{R}$.

TBB inhibits the effects of NaHS via phosphorylation of $A R C$

CK2 phosphorylates ARC at threonine-149 (Thr149), enabling ARC to translocate from the cytoplasm to the mitochondria (Li et al. 2002). Here we sought to determine whether blocking the activity of $\mathrm{CK} 2$ by its specific inhibitor, TBB, could inhibit the effects of NaHS on ARC phosphorylation. The cells were incubated in serum-free media containing vehicle (PBS), NaHS (400 $\mu \mathrm{mol} / \mathrm{L})$, or NaHS $(400 \mu \mathrm{mol} / \mathrm{L})+\mathrm{TBB}(5 \mu \mathrm{mol} / \mathrm{L})$ for $60 \mathrm{~min}$, and then subjected to $\mathrm{H} / \mathrm{R}$ as above. As shown in Fig. $4 \mathrm{~A}$ and $\mathrm{B}$, treatment of NaHS + TBB had little effect on the expression of 
A
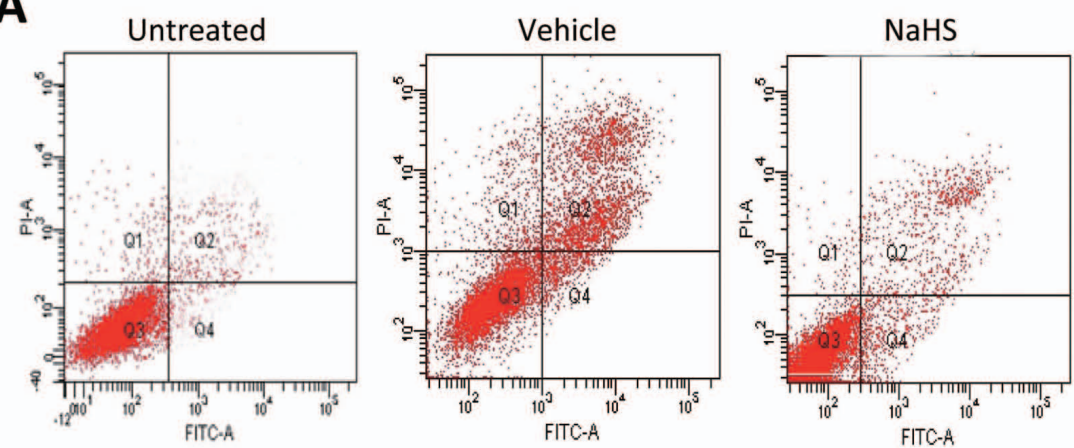

B
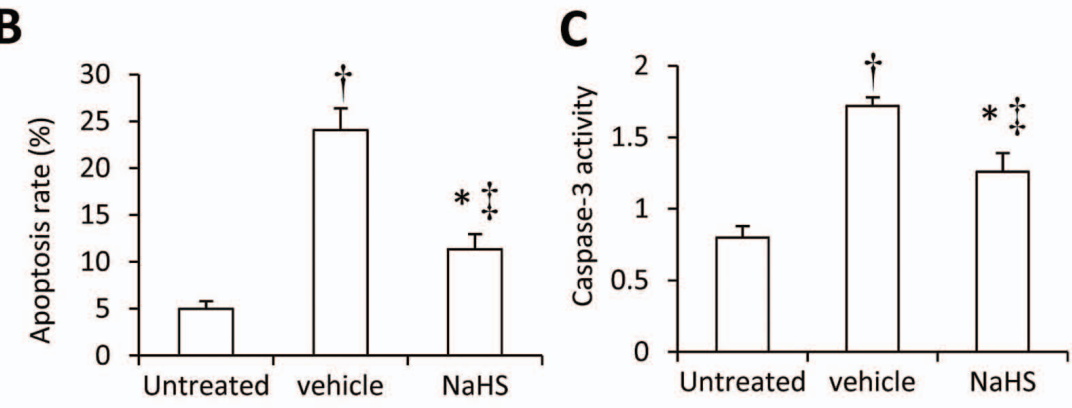

D
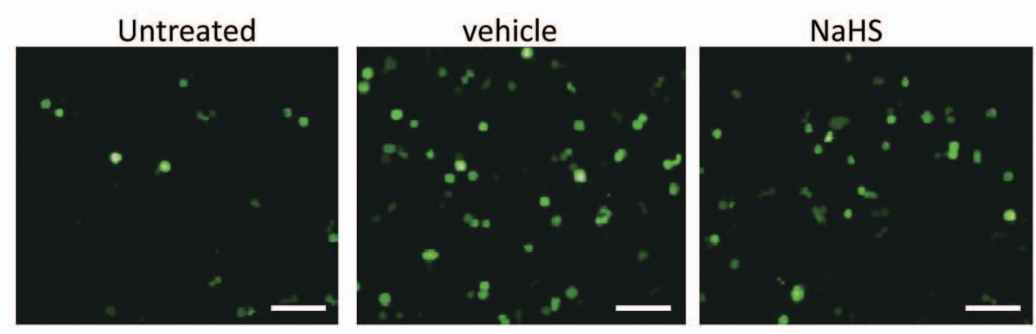

E

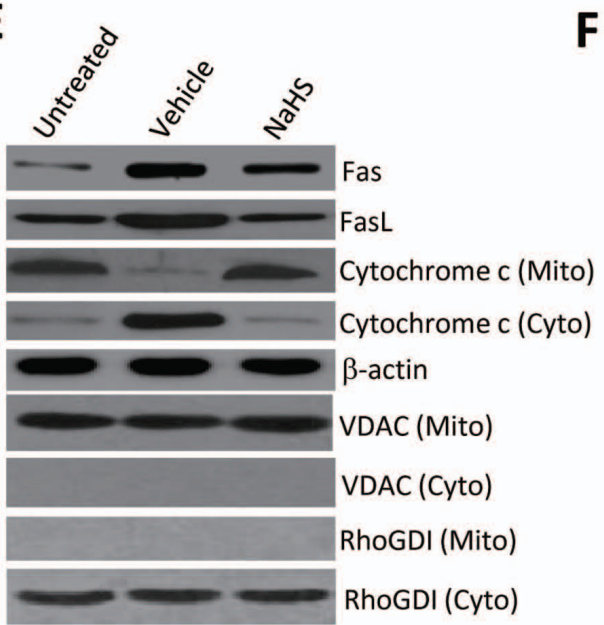

$\mathbf{F}$

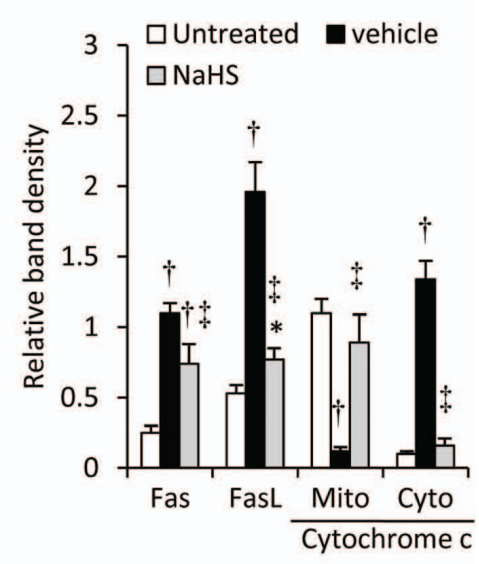

Fig. 2. Cell apoptosis. (A) The cells harvested as in Fig. 1 were stained with Annexin V and propidium iodide (PI). Representative dot plots were from cytometrically analyzed untreated cells, or cells subjected to $H / R$ and treated with vehicle or NaHS. The abscissa shows the number of Annexin V-positive cells, and the ordinate, PI-positive cells. (B) The apoptosis rates were plotted. (C) The activity of caspase-3 was measured. (D) The above cells were stained with TUNEL for detecting apoptotic cells. Bar $=50 \mu \mathrm{m}$. (E) The above cells were homogenized and Western blotted with Abs against Fas and FasL. The cytosolic (Cyto) and mitochondrial (Mito) factions were prepared, and cytochrome $c$ in both fractions was detected with Western blot analysis. The Abs against VDAC and RhoGDI were used to confirm the purity of mitochondrial and cytosolic fractions. (F) The density of each band was measured and compared to that of the internal control, $\beta$-actin. Compared to untreated cells, a significant difference is denoted by “*”, and a highly significant difference by " $\dagger$ " $(P<0.001)$. Compared to cells subjected to $\mathrm{H} / \mathrm{R}$ and treated vehicle, a significant difference is denoted by "it" $(P<0.05)$. 

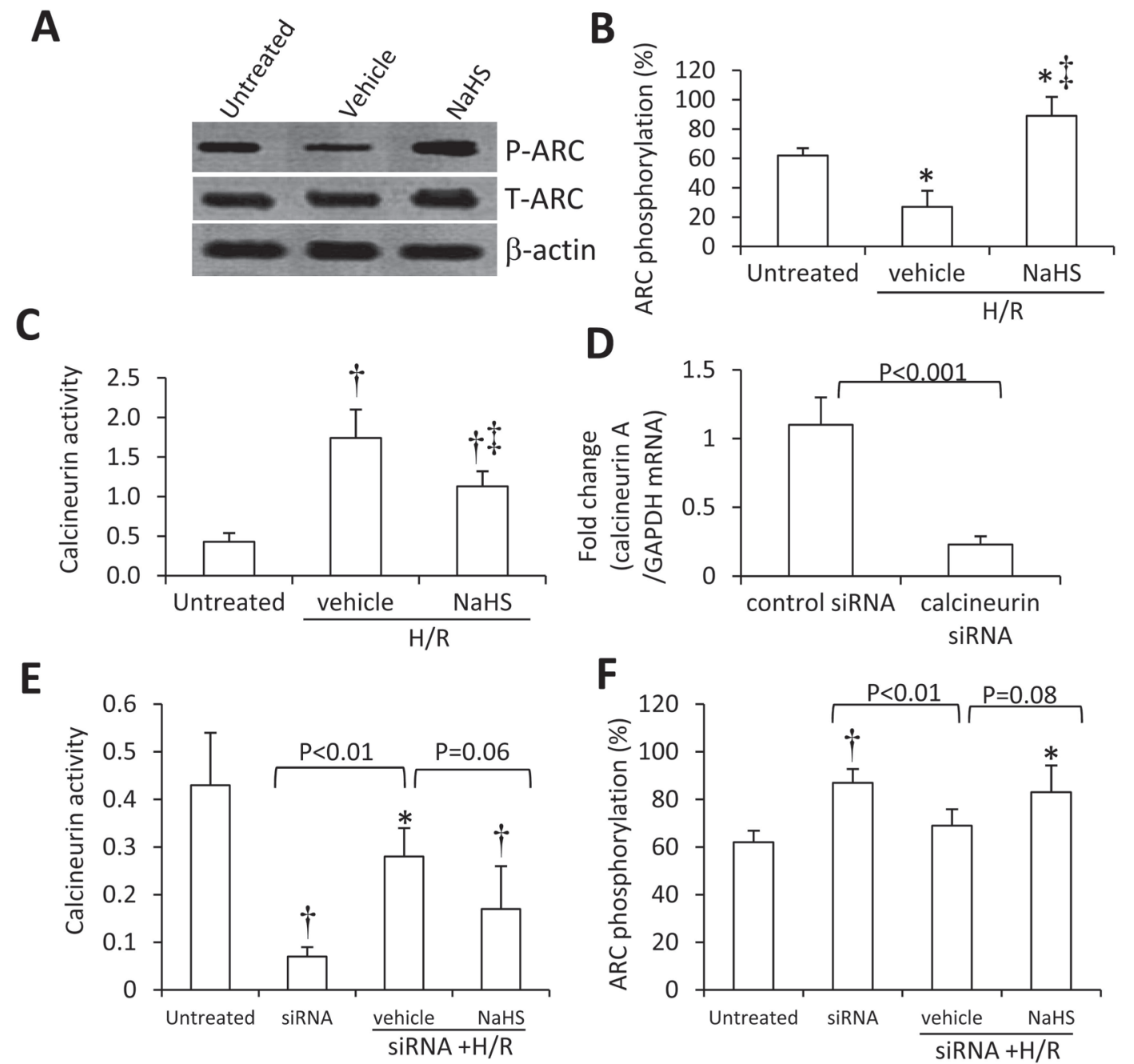

Fig. 3. NaHS enhances ARC phosphorylation by reducing calcineurin activity. (A) The cells were harvested as in Fig. 1, homogenized, and Western blotted with Abs against total ARC (T-ARC) or phosphorylated ARC (P-ARC). (B) The density of each band was measured to calculate ARC phosphorylation (\%). (C) The activity of calcineurin was measured. Compared to untreated cells, a significant difference is denoted by "*”, and a highly significant difference by "†" $(P<0.001)$. Compared to cells subjected to H/R and treated vehicle, a significant difference is denoted by " + ". (D) The cells were transfected with control or calcineurin siRNA, and the expression of calcineurin mRNA was measured and normalized with that of GAPDH. (E, F) The calcineurin activity (E) and ACR phosphorylation (\%) (F) in untreated cells, or calcineurin siRNA-transfected cells, or calcineurin siRNA-transfected cells exposed to $\mathrm{H} / \mathrm{R}$ and treated with vehicle or NaHS, were measured. Compared to untreated cells, a significant difference is denoted by “*”, and a highly significant difference by “†” $(P<0.001)$. $P$ values are shown above the two bars for comparison.

total ARC, but significantly $(P<0.05)$ reduced expression of phosphorylated ARC, compared with that treated with NaHS only. We next tested whether the effects of TBB on ARC phosphorylation could influence cell apoptosis and caspase-3 activity. As shown in Fig. $4 \mathrm{C}$ and D, treatment of $\mathrm{NaHS}+\mathrm{TBB}$ significantly increased both apoptosis rate and caspase- 3 activity of cells subjected to $H / R$, compared with that of cells treated with NaHS only $(P<0.05)$. In addition, we further demonstrated that NaHS significantly increased the activity of CK2, compared with the cells subjected to H/R and treated with vehicle (Fig. 4E).
NaHS reduces myocardial infarct size by inhibiting cell apoptosis through affecting ARC phosphorylation

First, we could show that the serum level of $\mathrm{H}_{2} \mathrm{~S}$ in MIR rats $(45.6 \pm 8.2 \mu \mathrm{M})$ was significantly $(P<0.05)$ higher than that in sham-operated rats $(19.7 \pm 3.4 \mu \mathrm{M})$, suggesting that MIR elevated $\mathrm{H}_{2} \mathrm{~S}$ levels in rats (Fig. 5A). Administration of NaHS significantly increased the level of $\mathrm{H}_{2} \mathrm{~S}(68.4 \pm 7.8 \mu \mathrm{M})$, compared with vehicle-treated MIR rats $(P<0.05)$. However, PAG further reduced $\mathrm{H}_{2} \mathrm{~S}$ level of $37.1 \pm 4.6 \mu \mathrm{M}$, which was highly significantly lower $(P$ $<0.001)$ than vehicle-treated MIR rats, but significantly higher than sham-operated rats $(P<0.05)$ (Fig. 5A).

As shown in Fig. 5B, there were almost no infarct-risk myocardial tissues observed in the sham-operated rats. 
A
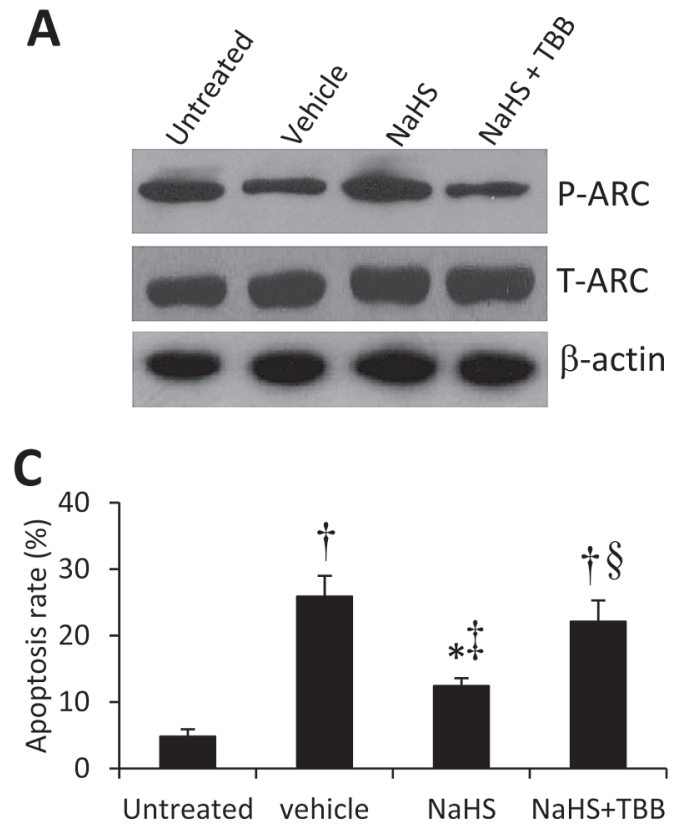

B

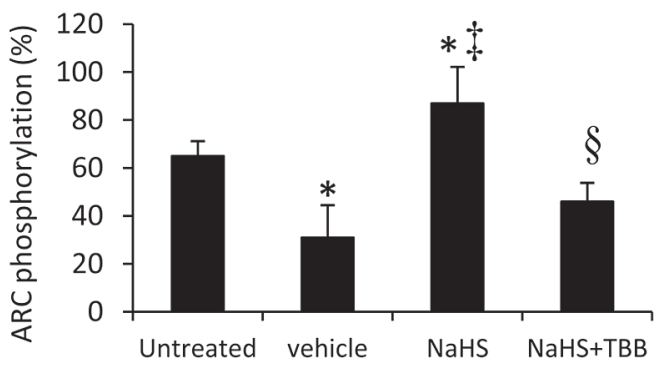

D

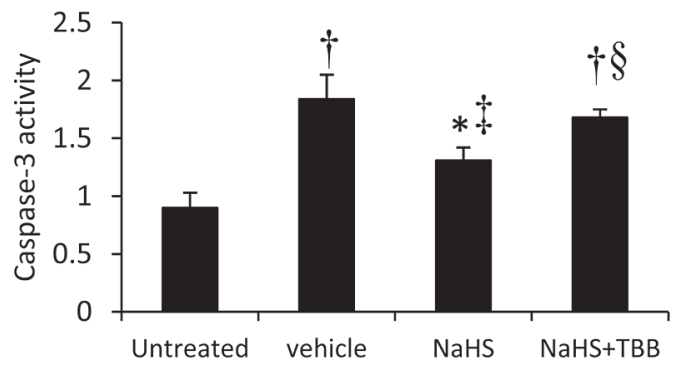

E

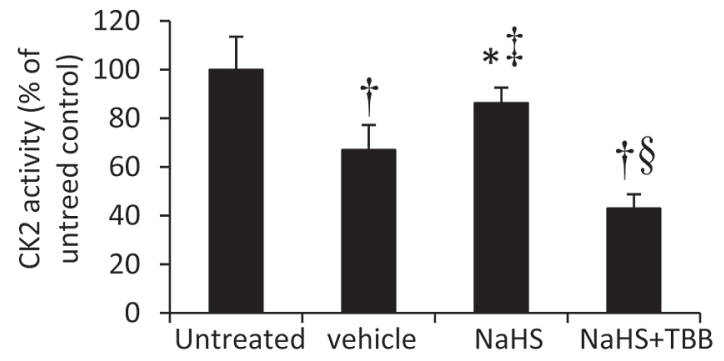

Fig. 4. TBB reversed the effects of NaHS on ARC phosphorylation and cell apoptosis. (A) Untreated cells, or cells subjected to $\mathrm{H} / \mathrm{R}$ and pre-incubated with vehicle, NaHS or NaHS + TBB were homogenized and Western blotted with Abs against total ARC (T-ARC) or phosphorylated ARC (P-ARC). (B-E) The ARC phosphorylation rate (B), apoptosis rate (C), caspase-3 activity (D) and CK2 activity were measured. Compared to untreated cells, a significant difference is denoted by “*”, and a highly significant difference by "†" $(P<0.001)$. Compared to cells subjected to H/R and treated vehicle, a significant difference is denoted by "\$”; and compared to cells subjected to H/R and treated with NaHS only, a significant difference by "§” ( $P<0.05)$.

MIR resulted in myocardial infarction with a mean infarct size of $41.6 \pm 6.1 \%$. Administration of NaHS significantly $(P<0.05)$ reduced the infarct size $(30.5 \pm 4.5 \%)$ compared to vehicle-treated MIR rats, whereas PAG further increased the infarct size $(56.8 \pm 7.3 \%)$, which was significantly greater than vehicle or NaHS-treated MIR rats $(P<0.05)$ (Fig. 5C).

MIR induced more apoptosis of myocardiocytes than sham operation, and administration of NaHS inhibited the apoptosis induced by MIR, whereas PAG further increased the apoptosis, compared with vehicle-treated MIR rats (Fig. 6A). The apoptosis index in the vehicle-treated MIR rats was significantly greater than sham-operated rats $(P<$ $0.001)$, NaHS significantly $(P<0.05)$ decreased the apoptosis index, while PAG significantly $(P<0.05)$ further increased the apoptosis index, compared with vehicletreated MIR rats (Fig. 6B).
In accordance with the results obtained in vitro, MIR significantly increased calcineurin activity, while administration of NaHS attenuated this increase and PAG further increased calcineurin activity in myocardial tissues (Fig. $6 \mathrm{C})$.

As shown in Fig. 6D and E, MIR significantly upregulated the expression levels of Fas, FasL, and cleaved caspase-3 proteins in myocardial tissues. However, administration of NaHS significantly attenuated the upregulation of Fas, FasL and cleaved caspase-3 $(P<0.05)$, while PAG further upregulated their expression already elevated by MIR. In addition, MIR significantly reduced ARC phosphorylation compared to sham-operated rats. NaHS significantly increased ARC phosphorylation, compared to vehicletreated MIR rats $(P<0.05)$, whereas administration of PAG further reduced ARC phosphorylation, compared to vehicletreated MIR rats (Fig. 6D and F). 


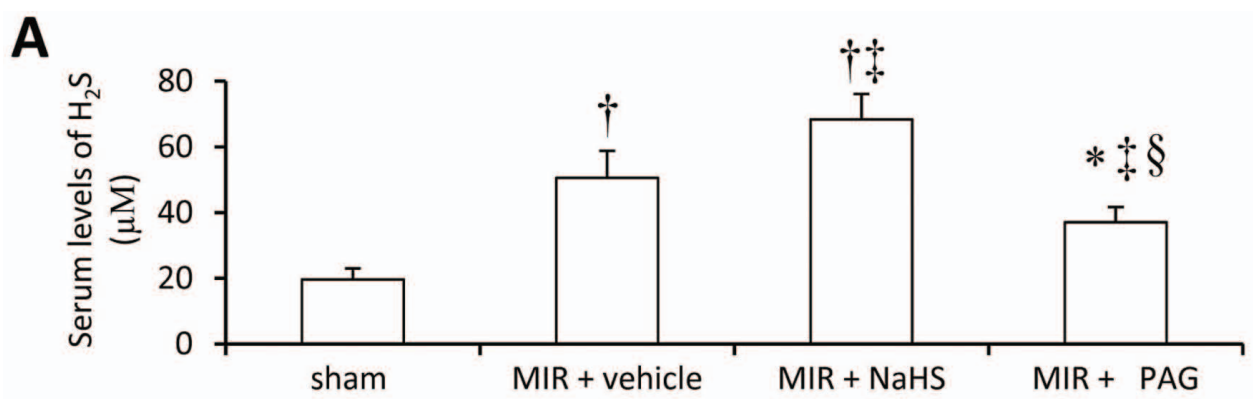

B

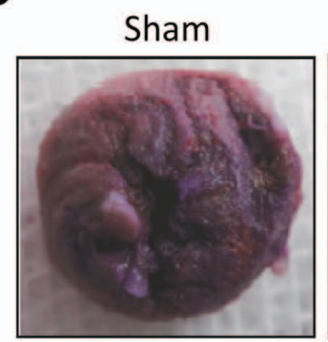

MIR+vehicle
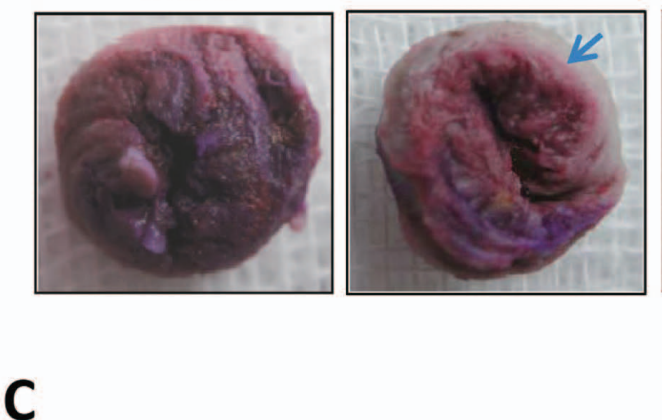

C

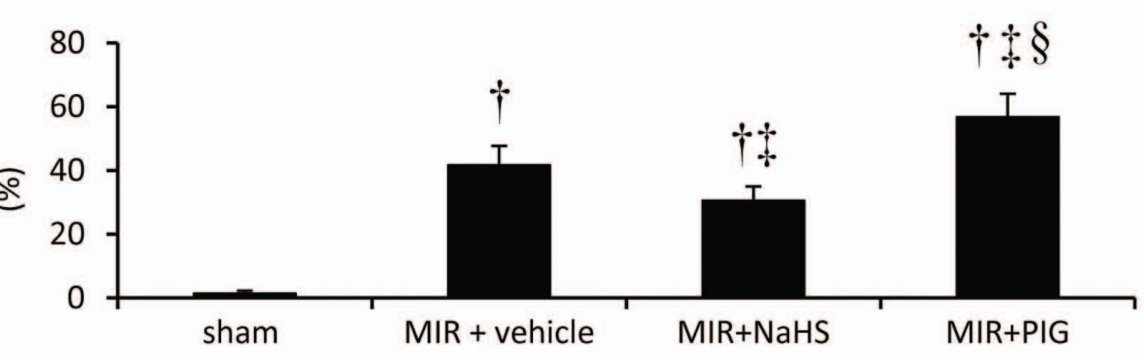

Fig. 5. NaHS reduces myocardial infarct size. Rats were subjected to sham operation, MIR, MIR+NaHS, or MIR+PAG. Blood samples and hearts were collected $2 \mathrm{~h}$ after ischemia. (A) The levels of $\mathrm{H}_{2} \mathrm{~S}$ in sera were measured. (B) Representative myocardial cross sections of TTC-stained hearts were taken each group. The brick red color indicates viable myocardium, while the infarct area appears pale (pointed by an arrow). A scale bar of $10 \mathrm{~mm}$ is shown. (C) The size of myocardial infarct was measured. Results are expressed as mean \pm S.D. $(n=6)$. Compared to sham-operated rats, a significant difference is denoted by “*”, a highly significant increase by " $\dagger$ " $(P<0.001)$. Compared to vehicle-treated MIR rats, a significant difference is denoted by "†"; and compared to NaHS-treated MIR rats, a significant difference by "§".

\section{Discussion}

MIR causes depressed myocardial function and deleterious morphological alterations, which may lead to heart failure (Wang et al. 2001). The mechanisms of MIRinduced injury include the production of reactive oxygen species, abnormal lipid metabolism and calcium overload (An et al. 2001). Apoptosis is thought to play an important role in MIR (Ekhterae et al. 2011). Therefore, agents that have anti-apoptotic activity may provide therapeutic potential to limit MIR-induced injury and heart failure. Here, we have demonstrated $\mathrm{H}_{2} \mathrm{~S}$ inhibited apoptosis of cardiomyocytes induced by MIR and $\mathrm{H} / \mathrm{R}$, in accordance with our previous study (Gao et al. 2011), and many other reports (Pan et al. 2006; Elrod et al. 2007; Bliksøen et al. 2008; Yong et al. 2008; Sivarajah et al. 2009). The elevated serum level of $\mathrm{H}_{2} \mathrm{~S}$ in MIR rats may be attributed to self-protective response of the host induced by MIR, in accordance with our previous report (Gao et al. 2011). Ischemia reperfusion increased the activation of caspase-3, release of cytochrome $c$ from mitochondria, and expression of Fas/FasL. However, NaHS significantly attenuated the above alterations, indicating that the effects of $\mathrm{H}_{2} \mathrm{~S}$ against MIRinduced injury may relies on both death receptor and mitochondrial apoptotic pathways, supported by previous reports (Pan et al. 2006; Elrod et al. 2007).

ARC is abundantly expressed in the heart, skeletal muscles and brains, and serves as an anti-apoptosis factor. ARC has been shown to block apoptotic cascades in hearts (Li et al. 2009). The anti-apoptotic activity of ARC depends on its phosphorylation state (Tan et al. 2008), which is a balance between the effects of CK2 and the dephosphorylation by calcineurin ( $\mathrm{Li}$ et al. 2002; Zhang and Herman 2006). It has been reported that ARC requires 


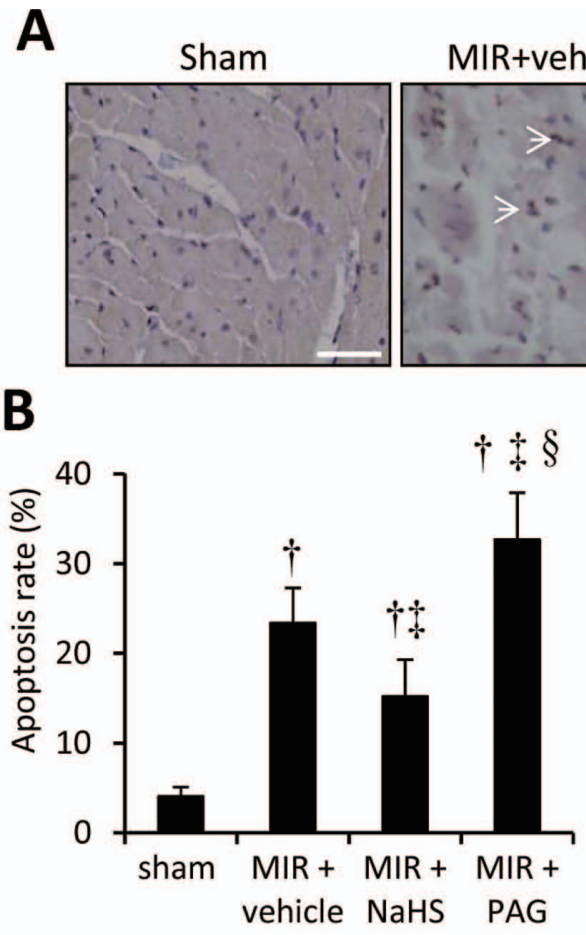

D

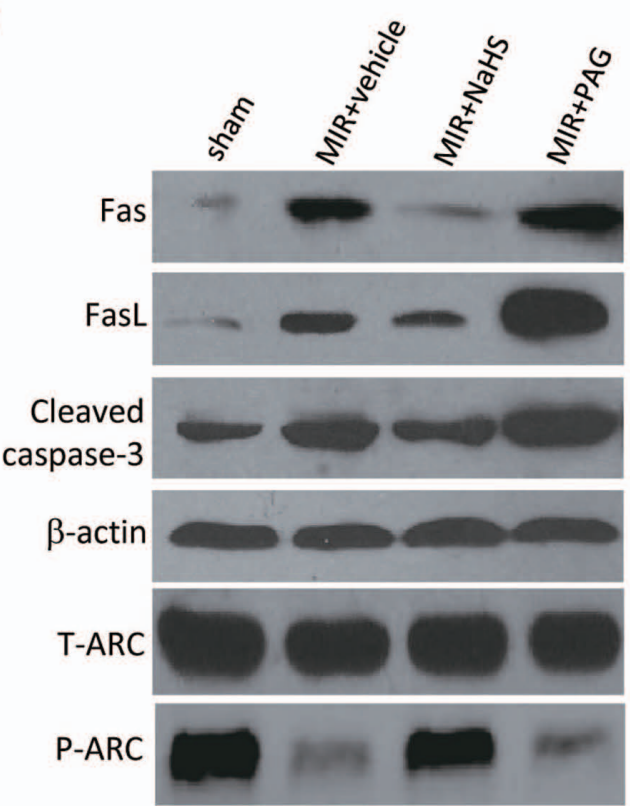

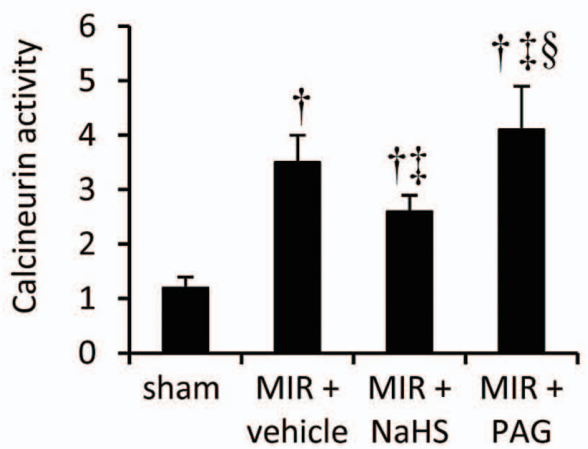

E

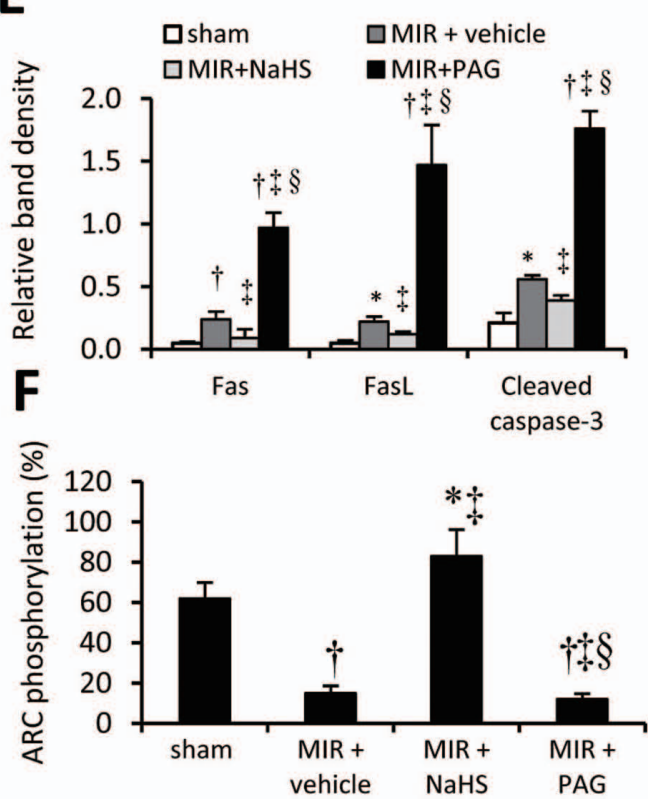

Fig. 6. Cell apoptosis, calcineurin activity and ARC phosphorylation in situ. (A) Representative photographs $(200 \times$ magnification) were taken from TUNEL-stained (TUNEL-positive cells are pointed by arrows) myocardial sections in rats in the sham, MIR + vehicle, MIR + NaHS and MIR + PAG groups. Bar $=100 \mu \mathrm{m}$. (B) TUNEL-positive cells were counted to record the apoptosis rate. (C) The activity of calcineurin in the myocardial tissues was measured. (D) Western blot analysis was used to detect expression of Fas, FasL, cleaved caspase-3, total ARC (T-ARC) and phosphorylated ARC (P-ARC) in myocardial homogenates from rats in the sham, MIR+vehicle, MIR+NaHS and MIR+PAG groups. (E) The density of each band was measured and compared to that of the internal control, $\beta$-actin. (F) The ARC phosphorylation rate (\%) was calculated. Results are expressed as mean \pm S.D. $(n=6)$. Compared to sham-operated rats, a significant difference is denoted by “*”, and a highly significant difference by " $†$ " $(P<0.001)$. Compared to vehicletreated MIR rats, a significant difference is denoted by " $\$$ ", and compared to NaHS-treated MIR rats, a significant difference by "§".

phosphorylation at Thr149 to protect cells against oxidative stress-induced apoptosis ( $\mathrm{Li}$ et al. 2002). Here, we have shown that either $\mathrm{H} / \mathrm{R}$ in vitro or MIR in vivo had little effect on the expression of total ARC, but significantly downregulated the expression of phosphorylated ARC, indicating the anti-apoptotic activity of ARC is inhibited by 
H/R and MIR. However, NaHS significantly increased ARC phosphorylation by increasing CK2 activity. To support this hypothesis, we have used TBB, a specific inhibitor of CK2, which is a key factor that phosphorylates ARC at Thr149. It has been reported that CK2-mediated ARC phosphorylation is constitutive, indicating the importance of phosphorylation for its function (Zhang and Herman 2006). Simultaneous treatment of TBB significantly reversed the effects of NaHS on ARC phosphorylation and $\mathrm{H} / \mathrm{R}$-induced cardiomyocyte apoptosis.

Calcium overload is one of the mechanisms of MIRinduced injury (An et al. 2001), and overloaded intracellular $\mathrm{Ca}^{2+}$ activates calcineurin, which accelerates the mitochondrial death signaling pathway (Wang et al. 1999). Here, we have shown that NaHS significantly reduced calcineurin activity, which was elevated by $\mathrm{H} / \mathrm{R}$. It has been reported that calcineurin dephosphorylates ARC in cardiomyocytes (Tan et al. 2008). To investigate whether NaHS increases ARC phosphorylation through its effects on calcineurin, calcineurin expression was knocked out by siRNA. The effects of NaHS on calcineurin activity and ARC phosphorylation were partially abolished in the calcineurinsilencing cells. In accord, $\mathrm{H}_{2} \mathrm{~S}$ preconditioning ameliorated intracellular $\mathrm{Ca}^{2+}$ handling in rat cardiomyocytes (Pan et al. 2008), and $\mathrm{H}_{2} \mathrm{~S}$ prevented $\mathrm{Ca}^{2+}$ overload in cardiomyocytes (Sivarajah et al. 2009). These results indicate that by reducing calcineurin activity, $\mathrm{H}_{2} \mathrm{~S}$ could inhibit dephosphorylation of ARC, and maintain phosphorylating status of ARC.

To further support the findings obtained in vitro, we tested the effects of $\mathrm{H}_{2} \mathrm{~S}$ in an in vivo MIR model by applying NaHS and PAG, an irreversible inhibitor of CSE. Administration of NaHS attenuated MIR-induced myocardial injury in rats, evidenced by the reduced myocardial infarct size, and inhibition of cell apoptosis. In support of the present study, $\mathrm{H}_{2} \mathrm{~S}$ was capable of inhibiting the progression of apoptosis by decreased activation of caspase-3 in a rat model of MIR (Zhao and Wang 2002), or by preserving mitochondrial function in a mouse model of MIR (Elrod et al. 2007). However, PAG further aggravated MIR-induced myocardial injury by decreasing serum levels of $\mathrm{H}_{2} \mathrm{~S}$. In consistence with the results obtained in vitro, NaHS attenuated the upregulation of Fas, FasL and cleaved caspase-3, the decreased ARC phosphorylation and increased calcineurin activity, induced by MIR. However, PAG further elevated expression of Fas, FasL and cleaved caspase-3, and reduced ARC phosphorylation.

In conclusion, the present study has for the first time demonstrated that $\mathrm{H}_{2} \mathrm{~S}$ inhibits apoptosis of cardiomyocytes induced by MIR through its effects on ARC phosphorylation. These results also indicate that targeting $\mathrm{H}_{2} \mathrm{~S}$ may present a promising approach against MIR-induced myocardial injury.

\section{Acknowledgments}

This work was supported by grants from the National Natural Scientific Foundation of China (30973474), and
Heilongjiang Provincial Scientific and Technology Bureau, China (QC06C075).

\section{Conflict of Interest}

All authors declare no conflict of interest.

\section{References}

An, J., Varadarajan, S.G., Camara, A., Chen, Q., Novalija, E., Gross, G.J. \& Stowe, D.F. (2001) Blocking $\mathrm{Na}(+) / \mathrm{H}(+)$ exchange reduces $[\mathrm{Na}(+)](\mathrm{i})$ and $[\mathrm{Ca}(2+)](\mathrm{i})$ load after ischemia and improves function in intact hearts. Am. J. Physiol. Heart Circ. Physiol., 281, H2398-H2409.

Bliksøen, M., Kaljusto, M.L., Vaage, J. \& Stensløkken, K.O. (2008) Effects of hydrogen sulphide on ischaemia-reperfusion injury and ischaemic pre-conditioning in the isolated, perfused rat heart. Eur. J. Cardiothorac. Surg., 34, 344-349.

Chen, P., Poddar, R., Tipa, E.V., Dibello, P.M., Moravec, C.D., Robinson, K., Green, R., Kruger, W.D., Garrow, T.A. \& Jacobsen, D.W. (1999) Homocysteine metabolism in cardiovascular cells and tissues: implications for hyperhomocysteinemia and cardiovascular disease. Adv. Enzyme Regul., 39, 93-109.

Donath, S., Li, P., Willenbockel, C., Al-Saadi, N., Gross, V., Willnow, T., Bader, M., Martin, U., Bauersachs, J., Wollert, K.C., Dietz, R., von Harsdorf, R. \& German Heart Failure Network. (2006) Apoptosis repressor with caspase recruitment domain is required for cardioprotection in response to biomechanical and ischemic stress. Circulation, 113, 12031212.

Ekhterae, D., Hinmon, R., Matsuzaki, K., Noma, M., Zhu, W., Xiao, R.P., Gorman, R.C. \& Gorman, J.H. 3rd. (2011) Infarction induced myocardial apoptosis and ARC activation. $J$. Surg. Res., 166, 59-67.

Ekhterae, D., Lin, Z., Lundberg, M.S., Crow, M.T., Brosius, 3rd. F.C. \& Nunez, G. (1999) ARC inhibits cytochrome $c$ release from mitochondria and protects against hypoxia-induced apoptosis in heart-derived H9c2 cells. Circ. Res., 85, e70-e77.

Elrod, J.W., Calvert, J.W., Morrison, J., Doeller, J.E., Kraus, D.W., Tao, L., Jiao, X., Scalia, R., Kiss, L., Szabo, C., Kimura, H., Chow, C.W. \& Lefer, D.J. (2007) Hydrogen sulfide attenuates myocardial ischemia-reperfusion injury by preservation of mitochondrial function. Proc. Natl. Acad. Sci. USA, 104, 15560-15565.

Fishbein, M.C., Meerabaum, S. \& Rit, J. (1981) Early phase acute myocardial infarct size quntification: Validation of the triphenyl tetrazolium chloride tissue enzyme staining technique. Am. Heart J., 101, 593-600.

Gao, Y., Yao, X., Zhang, Y., Li, W., Kang, K., Sun, L. \& Sun, X. (2011) The protective role of hydrogen sulfide in cardiac ischemia reperfusion-induced injury in diabetic rats. Int. J. Cardiol., 152, 177-183.

Gustafsson, A.B., Sayen, M.R., Williams, S.D., Crow, M.T. \& Gottlieb, R.A. (2002) TAT protein transduction into isolated perfused hearts: TAT-apoptosis repressor with caspase recruitment domain is cardioprotective. Circulation, 106, 735-739.

Hausenloy, D.J., Tsang, A., Mocanu, M.M. \& Yellon, D.M. (2005) Ischemic preconditioning protects by activating prosurvival kinases at reperfusion. Am. J. Physiol. Heart Circ. Physiol., 288, H971-H976.

Jo, D.G., Jun, J.I., Chang, J.W., Hong, Y.M., Song, S., Cho, D.H., Shim, S.M., Lee, H.J., Cho, C., Kim, D.H. \& Jung, Y.K. (2004) Calcium binding of ARC mediates regulation of Caspase 8 and cell death. Mol. Cell Biol., 24, 9763-9770.

Kang, K., Zhao, M., Jiang, H., Tan, G. \& Sun, X. (2009) The role of hydrogen sulfide in hepatic ischemia reperfusion-induced injury in rats. Liver Transpl., 15, 1306-1314.

Li, P.F., Li, J., Muller, E.C., Otto, A., Dietz, R. \& von Harsdorf, R. 
(2002) Phosphorylation by protein kinase CK2: a signaling switch for the caspase-inhibiting protein ARC. Mol. Cell, 10, 247-258.

Li, Y., Ge, X. \& Liu, X. (2009) The cardioprotective effect of postconditioning is mediated by ARC through inhibiting mitochondrial apoptotic pathway. Apoptosis, 14, 164-172.

Li, Y.Z., Liu, X.H., Zhu, X.M. \& Cai, L.R. (2007) ARC contributes to the inhibitory effect of preconditioning on cardiomyocyte apoptosis. Apoptosis, 12, 1589-1595.

£owicka, E. \& Betowski, J. (2007) Hydrogen sulfide (H2S) - the third gas of interest for pharmacologists. Pharmacol. Rep., 59, 4-24.

Lu, X., Moore, P.G., Liu, H. \& Schaefer, S. (2011) Phosphorylation of ARC is a critical element in antiapoptotic effect of anesthetic preconditioning. Anesth. Analg., 112, 525-531.

Nam, Y.J., Mani, K., Ashton, A.W., Peng, C.F., Krishnamurthy, B., Hayakawa, Y., Lee, P., Korsmeyer, S.J. \& Kitsis, R.N. (2004) Inhibition of both the extrinsic and intrinsic death pathways through nonhomotypic death-foldinteractions. Mol. Cell, 15, 901-912.

Pan, T.T., Feng, Z.N., Lee, S.W., Moore, P.K. \& Bian, J.S. (2006) Endogenous hydrogen sulfide eontributes to the cardioprotection by metabolic inhibition preconditioning in the rat venirieular myoeytes. J. Mol. Cell Cardiol., 40, 119-130.

Pan, T.T., Neo, K.L., Hu, L.F., Yong, Q.C. \& Bian, J.S. (2008) $\mathrm{H}_{2} \mathrm{~S}$ preconditioning-induced $\mathrm{PKC}$ activation regulates intracellular calcium handling in rat cardiomyocytes. Am. J. Physiol. Cell Physiol., 294, C169-C177.

Prasad, A.M. \& Inesi, G. (2011) Silencing calcineurin A subunit reduces SERCA2 expression in cardiac myocytes. Am. J. Physiol. Heart Circ. Physiol., 300, H173-180.

Shiraishi, J., Tatsumi, T., Keira, N., Akashi, K., Mano, A., Yamanaka, S., Matoba, S., Asayama, J., Yaoi, T., Fushiki, S., Fliss, H. \& Nakagawa, M. (2001) Important role of energydependent mitochondrial pathways in cultured rat cardiac myocyte apoptosis. Am. J. Physiol. Heart Circ. Physiol., 281, H1637-H1647.

Sivarajah, A., Collino, M., Yasin, M., Benetti, E., Gallicchio, M.,
Mazzon, E., Cuzzocrea, S., Fantozzi, R. \& Thiemermann, C. (2009) Anti-apoptotic and anti-inflammatory effects of hydrogen sulfide in a rat model of regional myocardial I/R. Shock, 31, 267-274.

Sivarajah, A., McDonald, M.C. \& Thiemermann, C. (2006) The production of hydrogen sulfide limits myocardial ischemia and reperfusion injury and contributes to the cardioprotective effects of preconditioning with endotoxin, but not ischemia in the rat. Shock, 26, 154-161.

Tan, W.Q., Wang, J.X., Lin, Z.Q., Li, Y.R., Lin, Y. \& Li, P.F. (2008) Novel cardiac apoptotic pathway: the dephosphorylation of apoptosis repressor with caspase recruitment domain by calcineurin. Circulation, 118, 2268-2276.

Wang, G.W., Zhou, Z., Klein, J.B. \& Kang, Y.J. (2001) Inhibition of hypoxia/reoxygenation-induced apoptosis in metallothionein-overexpressing cardiomyocytes. Am. J. Physiol. Heart Circ. Physiol., 280, H2292-H2299.

Wang, H.G., Pathan, N., Ethell, I.M., Krajewski, S., Yamaguchi, Y., Shibasaki, F., McKeon, F., Bobo, T., Franke, T.F. \& Reed, J.C. (1999) Ca2+-Induced apoptosis through calcineurin dephosphorylation of BAD. Science, 284, 339-343.

Yao, L.L., Huang, X.W., Wang, Y.G., Cao, Y.X., Zhang, C.C. \& Zhu, Y.C. (2010) Hydrogen sulfide protects cardiomyocytes from hypoxia/reoxygenation-induced apoptosis by preventing GSK-3beta-dependent opening of mPTP. Am. J. Physiol. Heart Circ. Physiol., 298, H1310-H1319.

Yong, Q.C., Lee, S.W., Foo, C.S., Neo, K.L., Chen, X. \& Bian, J.S. (2008) Endogenous hydrogen sulphide mediates the cardioprotection induced by ischemic postconditioning. Am. J. Physiol. Heart Circ. Physiol., 295, H1330-H1340.

Zhang, Y.Q. \& Herman, B. (2006) ARC protects rat cardiomyocytes against oxidative stress through inhibition of caspase-2 mediated mitochondrial pathway. J. Cell Biochem., 99, $575-588$.

Zhao, W.M. \& Wang, R. (2002) H2S-induced vasorelaxation and underlying cellular and molecular mechanisms. Am. J. Physiol. Heart Circ. Physiol., 283, H474-H480. 